\title{
Dark matter from hidden forces
}

\author{
Hooman Davoudiasl and Ian M. Lewis \\ Department of Physics, Brookhaven National Laboratory, Upton, New York 11973, USA
}

(Received 11 October 2013; published 25 March 2014)

\begin{abstract}
We examine the possibility that dark matter may be the manifestation of dark forces of a hidden sector, i.e. "dark force $=$ dark matter." As an illustrative and minimal example we consider the hidden $\mathrm{SU}(2)_{h} \times$ $\mathrm{U}(1)_{h}$ gauge group. The hidden dynamics is indirectly coupled to the Standard Model (SM) through kinetic mixing of $\mathrm{U}(1)_{h}$ with the $\mathrm{U}(1)_{Y}$ of hypercharge. We assume a hidden symmetry-breaking pattern analogous to that of the SM electroweak symmetry, augmented with an extra scalar that allows both the "hidden $Z$ boson" $Z_{h}$ and the "hidden photon" $\gamma_{h}$ to be massive. The "hidden $W$ " bosons $W_{h}^{ \pm}$are dark matter in this scenario. This generic setup can readily accommodate potential direct detection signals for light dark matter. For some choices of parameters, the model can lead to signals both in "dark matter beam" experiments, from $Z_{h} \rightarrow W_{h} W_{h}$, as well as in experiments that look for visible signals of dark photons, mediated by $\gamma_{h}$. Other possible phenomenological consequences are also briefly discussed.
\end{abstract}

DOI: $10.1103 /$ PhysRevD.89.055026

PACS numbers: 95.35.+d, 12.60.-i

\section{INTRODUCTION}

The nature of dark matter (DM) remains a mystery. Observational evidence based on its gravitational effects suggests that DM makes up roughly $25 \%$ of the cosmic energy density [1] and does not have any significant nongravitational interactions with ordinary matter. This more or less sums up our understanding of DM which is why there are a variety of currently viable ideas for describing it. So far, there is no clear signal of DM, but there are tentative hints in various experiments, both astrophysical and terrestrial. Some astrophysical data [2-4] may be accommodated by DM models that contain new light vector bosons that mediate dark-sector forces with feeble couplings to the visible Standard Model (SM) sector [5]. Of the laboratory signals, some hint at a potential signal for light DM around $\sim 10 \mathrm{GeV}$ [6-10].

Whether any of the aforementioned hints will grow in significance and rise to the level of a clear discovery remains to be seen. Nonetheless, speculative thinking on this subject has led to new models of DM, some of which postulate a dark sector endowed with its own interactions, i.e. "dark forces," characterized by scales near $1 \mathrm{GeV}$. Many of these models use fermion or scalar DM candidates with an $a d$ hoc parity to guarantee stability. However, if we use the SM as a guide, the stability of particles is expected to be derived from gauge symmetries, Lorentz symmetry, or accidental symmetries that are a result of the gauge and Lorentz symmetries [11].

If the dark sector is endowed with a gauge symmetry, it may be possible to assume that DM is made up of dark gauge fields whose mass has been generated by a Higgs mechanism. This possibility arises in cases where, after symmetry breaking, the non-Abelian gauge bosons are stable due to residual symmetries, obviating the need for additional fermions or scalars. Such minimal scenarioswhich imply "dark force $=$ dark matter" - have garnered interest recently $[12,13]$. However, these models typically rely on a Higgs portal to communicate with the SM and obtain the correct relic abundance. ${ }^{1}$ Here we point out that if we augment a non-Abelian hidden gauge symmetry with an additional $\mathrm{U}(1)_{h}$, the dark sector and $\mathrm{SM}$ can interact via kinetic mixing between the $\mathrm{U}(1)_{h}$ gauge boson and the SM hypercharge gauge boson. Such a scenario can also lead to a rich phenomenology in low-energy experiments [14-35] if: as indicated by CDMSII-Si data, the dark sector lives in the mass range $\lesssim \mathcal{O}(10) \mathrm{GeV}$. For additional proposals with spin-1 DM candidates originating from other sources see Refs. [36-38].

\section{THE MODEL}

To show the viability of our scenario, we focus on the minimal Higgsed gauge group with a non-Abelian symmetry and kinetic mixing with the SM. That is, we will assume a hidden gauge group $\mathrm{SU}(2)_{h} \times \mathrm{U}(1)_{h}$ (i.e. a gauge group that the SM is uncharged under). The hidden gauge symmetries are broken in close analogy with the electroweak symmetry of the SM. In particular, we will assume a hidden Higgs doublet $\Phi_{h}$ of $\mathrm{SU}(2)_{h}$ with charge $1 / 2$ under $\mathrm{U}(1)_{h}$, leading to massive hidden vectors $W_{h}^{ \pm}$by developing a nonzero vacuum expectation value $\left\langle\Phi_{h}\right\rangle=\left(0, v_{\Phi}\right)^{\mathrm{T}} / \sqrt{2}$. In order to break the remaining $\mathrm{U}(1)$, thereby giving mass to the "hidden" photon, we also introduce a complex scalar $\phi_{h}$ with charge $1 / 2$ only under $\mathrm{U}(1)_{h}$ and $\left\langle\phi_{h}\right\rangle=v_{\phi} / \sqrt{2}$. In the following, $g_{h}$ and $g_{h}^{\prime}$ will denote the $\mathrm{SU}(2)_{h}$ and $\mathrm{U}(1)_{h}$ gauge couplings, respectively.

\footnotetext{
${ }^{1}$ We note that Ref. [13] assumes a $\mathrm{U}(1)_{B-L}$ gauge symmetry as part of the dark vector bosons to induce direct coupling to SM particles.
} 
We have

$$
W_{h \mu}^{ \pm}=\frac{1}{\sqrt{2}}\left(W_{h \mu}^{1} \pm i W_{h \mu}^{2}\right)
$$

where $W_{h \mu}^{i}$, with $i=1,2,3$, are the $\mathrm{SU}(2)_{h}$ gauge fields and \pm refers to "hidden electric charges." The masses of $W_{h}^{ \pm}$are given by the usual SM relation adapted to the hidden sector,

$$
M_{W_{h}}=\frac{g_{h}}{2} v_{\Phi}
$$

The vacuum expectation value (VEV) of the Higgs doublet, $v_{\Phi}$, leads to mixing between the other "neutral" vector bosons (analogues of the SM $Z$ and photon) parametrized by an angle $\theta_{h}$. Denoting these mass eigenstates by $Z_{h}$ and $\gamma_{h}$, we have

$$
Z_{h \mu}=\cos \theta_{h} W_{h \mu}^{3}-\sin \theta_{h} B_{h \mu}
$$

and

$$
\gamma_{h \mu}=\sin \theta_{h} W_{h \mu}^{3}+\cos \theta_{h} B_{h \mu},
$$

where $B_{h \mu}$ is the gauge field associated with $\mathrm{U}(1)_{h}$. One can show

$$
\cos ^{2} \theta_{h}=\frac{M_{W_{h}}^{2}-M_{\gamma_{h}}^{2}}{M_{Z_{h}}^{2}-M_{\gamma_{h}}^{2}}
$$

where $M_{W_{h}}, M_{Z_{h}}$, and $M_{\gamma_{h}}$ denote the masses of $W_{h}, Z_{h}$, and $\gamma_{h}$, respectively. We will provide expressions for these masses in the Appendix. Note that in the limit $M_{\gamma_{h}} \rightarrow 0$ we recover the SM-like relation $M_{W_{h}}^{2}=\cos ^{2} \theta_{h} M_{Z_{h}}^{2}$ (with $\theta_{h}$ being the analogue of the weak mixing angle $\theta_{W}$ ).

While the SM fields do not carry charges under $\mathrm{SU}(2)_{h} \times \mathrm{U}(1)_{h}$, the two sectors are assumed to be coupled through a renormalizable kinetic mixing term [39-41]

$$
\frac{\varepsilon}{2 \cos \theta_{W}} B_{h}^{\mu \nu} B_{\mu \nu}
$$

where $X_{\mu \nu}=\partial_{\mu} X_{\nu}-\partial_{\nu} X_{\mu}$, and $B_{\mu}$ is the SM hypercharge gauge field. For simplicity we assume that kinetic mixing is the only portal between the dark sector and the SM, and ignore possible mixing in the Higgs sector. Additionally, the mixing between the SM and dark-sector Higgs bosons may be expected to be small due to fine-tuning arguments, as discussed in the next section.

Upon diagonalization of the kinetic terms in the usual way (see for example Ref. [35] and the Appendix), one finds that the two massive vectors, $\gamma_{h}$ and $Z_{h}$, couple to the visible electromagnetic current $J_{\mathrm{em}}^{\mu}$ according to

$$
\mathcal{L}_{\mathrm{vh}}=-\varepsilon e\left[\cos \theta_{h} \gamma_{h \mu}-\sin \theta_{h} Z_{h \mu}\right] J_{\mathrm{em}}^{\mu},
$$

where $e$ is the SM electromagnetic coupling.

\section{RELIC DENSITY}

For $W_{h}$ to be viable as a dark matter candidate, it needs to be cosmologically stable, have the correct relic density $\Omega_{\mathrm{DM}}$, and not be ruled out by direct or indirect searches. With the above assumptions about the hidden sector, the $W_{h}$ vectors are stable particles and will not decay. This is due to a remnant $Z_{2}$ that persists after $\mathrm{SU}(2)_{h} \times \mathrm{U}(1)_{h}$ breaking in this scenario. ${ }^{2}$ To answer the other questions, we need to calculate the thermally averaged annihilation cross section $\left\langle\sigma_{\text {ann }} v_{\text {rel }}\right\rangle$, with $v_{\text {rel }}$ the relative velocity, and the elastic scattering cross section $\sigma_{\mathrm{el}}$ from nucleons, which in our case only include protons.

For the simplicity of our treatment, while maintaining the key aspects of the model, we will henceforth assume $M_{\gamma_{h}} \ll M_{W_{h}}$ with $\eta \equiv v_{\phi} / v_{\Phi} \ll 1$, and $M_{Z_{h}}, M_{\Phi}>2 M_{W_{h}}$, where $M_{\Phi}$ is the mass of $\Phi$. Then, the dominant process that would set the relic density of $W_{h}$ is $W_{h} W_{h} \rightarrow \gamma_{h} \gamma_{h}$, given by the Feynman diagrams in Fig. 1. The $s$-channel annihilation through $\Phi$ is suppressed by the small $\Phi \gamma_{h} \gamma_{h}$ coupling which is proportional to $\eta^{4}$. This suppression does not apply to $\phi \gamma_{h} \gamma_{h}$ coupling and one has to consider the effect of $\Phi-\phi$ mixing in the $s$-channel diagram. The scalar mixing will come from a term $\lambda_{m} \phi^{\dagger} \phi \Phi^{\dagger} \Phi$. For a perturbative self-coupling of $\phi$, we then expect $\mu_{\phi} \lesssim v_{\phi}$, where $\mu_{\phi}$ is the mass parameter in the $\phi$ potential. Hence, unless $\mu_{\phi}$ is set by a tuned cancellation, we must assume $\lambda_{m} v_{\Phi}^{2} \lesssim v_{\phi}^{2}$ which yields $\lambda_{m} \lesssim \eta^{2}$. Thus, $\Phi-\varphi$ mixing is typically suppressed by $\eta^{2}{ }^{3}$. We note that these arguments can be theoretically justified if our setup is viewed as an effective description that descends from a high-scale theory, where the field content and symmetries can result in the assumed suppression of scalar mixing terms. However, such ultraviolet model building is beyond the scope of our treatment.

In the following discussion, we will implicitly assume that DM and the SM sector are in thermal equilibrium before freeze-out, and are hence at the same temperature. We will address this point later in this section. Since $\eta \ll 1$, it is expected that $M_{\phi}<M_{W_{h}}$ and one may consider the annihilation channel $W_{h} W_{h} \rightarrow \phi \phi$. Since $\phi$ is an $\mathrm{SU}(2)_{h}$ singlet, its coupling to $W_{h}$ will proceed through $\Phi-\varphi$ mixing. Using the arguments presented above, the annihilation cross section for $W_{h} W_{h} \rightarrow \phi \phi$ scales as $g_{h}^{2} \eta^{4}$, doubly suppressed by $\Phi-\varphi$ mixing. This should be compared to the

\footnotetext{
${ }^{2}$ The effective operator $\left(\phi D_{\mu} \phi\right)^{*}\left(\Phi D^{\mu} \Phi\right)$ can mix $W_{h}^{ \pm}$with $B_{h}$, generating decays of $W_{h}^{ \pm}$to SM fermions. To be a viable DM candidate, $W_{h}^{ \pm}$needs a lifetime of $\sim 10^{27}$ seconds [42]. With the assumptions in this paper, we find that this operator needs to be suppressed by a mass scale $\gtrsim 10^{12} \mathrm{GeV}$.

${ }^{3}$ Similar fine-tuning arguments can also be made to show that the mixing between the SM and dark-sector Higgs bosons are expected to be suppressed by powers of $v_{\phi, \Phi} / v_{\mathrm{EW}}$.
} 

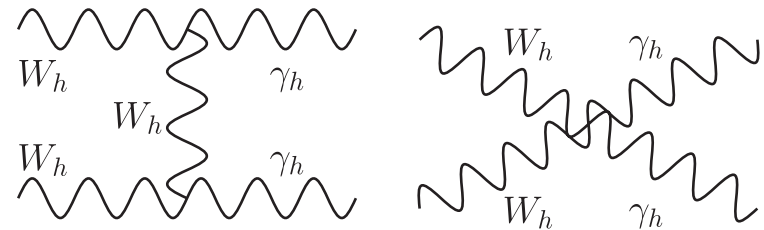

FIG. 1. Leading annihilation process within the set of simplifying assumptions in the text. There is also a third annihilation diagram that is obtained from the left one by a crossing.

$W_{h} W_{h} \rightarrow \gamma_{h} \gamma_{h}$ cross section scaling as $g_{h}^{2}$. Hence, $W_{h} W_{h} \rightarrow \gamma_{h} \gamma_{h}$ is the dominant annihilation channel.

These processes are governed by the $W_{h} W_{h} \gamma_{h}$ and $W_{h} W_{h} \gamma_{h} \gamma_{h}$ vertices whose Lorentz structure is identical to the familiar analogues in the SM. However, the overall coupling here is set by $g_{h} \sin \theta_{h}$. We find that the thermally averaged annihilation cross section is well approximated by

$$
\left\langle\sigma_{\mathrm{ann}} v_{\mathrm{rel}}\right\rangle \simeq \frac{19\left(g_{h} \sin \theta_{h}\right)^{4}}{72 \pi M_{W_{h}}^{2}},
$$

where the mass of $\gamma_{h}$ has been ignored. More detailed results, including the $p$-wave contributions, are given in the Appendix and are used to obtain our numerical results presented below. The relic density of $W_{h}$ is given by [43]

$$
\Omega_{h} h^{2} \simeq 1.04 \times 10^{9} \frac{x_{f} \mathrm{GeV}^{-1}}{\sqrt{g_{\star}} M_{\mathrm{Pl}}\left\langle\sigma_{\mathrm{ann}} v_{\mathrm{rel}}\right\rangle},
$$

where $g_{\star}$ is the number of relativistic degrees of freedom at the time of freeze-out and $M_{\mathrm{Pl}} \simeq 1.22 \times 10^{19} \mathrm{GeV}$ is the Planck mass. The quantity $x_{f}=M_{W_{h}} / T_{f}$, with $T_{f}$ the freeze-out temperature, is given by [43]

$$
x_{f} \simeq \ln \left[0.038\left(\kappa / \sqrt{x_{f} g_{\star}}\right) M_{\mathrm{Pl}} M_{W_{h}}\left\langle\sigma_{\mathrm{ann}} v_{\text {rel }}\right\rangle\right],
$$

where $\kappa=3$ for a massive vector boson. The insertion of $\left\langle\sigma_{\text {ann }} v_{\text {rel }}\right\rangle$ in Eqs. (9) and (10) is valid for only the $s$-wave approximation. A more complete expression for the relic density, including $p$-wave contributions, is given in the Appendix. For the range of parameters relevant in our work $x_{f} \simeq 20$ is a good approximation and we will use this value in the following.

If dark matter is composed only of $W_{h}$, then the relic density $\Omega_{h} h^{2} \simeq 0.12$ [1] can be used to solve for $g_{h} \sin \theta_{h}$ in terms of $M_{W_{h}}$. Under this assumption and $M_{\gamma_{h}} \ll M_{W_{h}}$, we find

$$
\left(g_{h} \sin \theta_{h}\right)^{2} \simeq \frac{M_{W_{h}}}{10 \mathrm{GeV}} \begin{cases}2.2 \times 10^{-3} ; & T_{f} \lesssim \Lambda_{\mathrm{QCD}}, \\ 1.5 \times 10^{-3} ; & T_{f} \gtrsim \Lambda_{\mathrm{QCD}},\end{cases}
$$

where the $p$-wave expansion and $x_{f} \simeq 20$ have been used. The two solutions are due to the different counting of degrees of freedom below and above the QCD phase transition, which we assume to occur at $\Lambda_{\mathrm{QCD}} \simeq 200 \mathrm{MeV}$.
For freeze-out temperatures below the QCD phase transition we use $g_{\star}=13.75$ accounting for the neutrinos, electron, photon, and $\gamma_{h}$; for freeze-out temperatures above the QCD phase transition we additionally include the muon, gluons, and $u, d, s$ quarks in the counting and find $g_{\star}=64.75$. While the inclusion of $\gamma_{h}$ in $g_{\star}$ depends critically on $M_{\gamma_{h}}$ and charm quarks (muons) should be included for $T_{f} \gtrsim M_{c}\left(\Lambda_{\mathrm{QCD}} \gtrsim T_{f} \gtrsim M_{\mu}\right)$, we find that these considerations only make $\sim 5 \%$ corrections in our determination of $\left(g_{h} \sin \theta_{h}\right)^{2}$. Hence, for simplicity we neglect these effects in the numerical results that follow.

As mentioned before, an implicit assumption in the above derivation was that dark matter initially starts in thermal equilibrium before freeze-out. For consistency, we would then require that the hidden photon $\gamma_{h}$ decay rate $\Gamma_{\gamma_{h}}$ into SM final states is large enough to keep up with the expansion of the Universe at $T=T_{f}$ [43]. Assuming $M_{\gamma_{h}} \lesssim 1 \mathrm{GeV}$, the decay rate is

$$
\begin{aligned}
\Gamma_{\gamma_{h}} & \simeq \frac{\alpha}{3}\left(\varepsilon \cos \theta_{h}\right)^{2} M_{\gamma_{h}} \sum_{F} N_{C} Q_{F}^{2}\left(1+\frac{2 M_{F}^{2}}{M_{\gamma_{h}}^{2}}\right) \beta_{F} \\
& \lesssim \frac{4 \alpha}{3}\left(\varepsilon \cos \theta_{h}\right)^{2} M_{\gamma_{h}},
\end{aligned}
$$

where $\alpha \equiv e^{2} /(4 \pi) \simeq 1 / 137, \beta_{F}=\sqrt{1-4 M_{F}^{2} / M_{\gamma_{h}}^{2}}$, and the sum is over fermions, $F$, with masses $2 M_{F} \leq M_{\gamma_{h}}$, charges $Q_{F}$, and colors $N_{C}=1$ for leptons and $N_{C}=3$ for quarks. The inequality is obtained for $F \in\{e, \mu, u, d, s\}$. The expansion rate at freeze-out is set by the Hubble constant $H\left(T_{f}\right)=1.7 g_{\star}^{1 / 2} T_{f}^{2} / M_{\mathrm{Pl}}$. For $M_{\gamma_{h}}<T_{f}$ the requirement that $\gamma_{h}$ are in thermal equilibrium at $T_{f}$ is satisfied when [43]

$$
\frac{M_{\gamma_{h}}}{T_{f}} \Gamma_{\gamma_{h}} \gtrsim H\left(T_{f}\right) .
$$

Using this condition and $T_{f}=M_{W_{h}} / 20$ yields

$$
\left(\varepsilon \cos \theta_{h}\right)^{2}\left(\frac{M_{\gamma_{h}}}{\mathrm{MeV}}\right)^{2} \gtrsim 10^{-12} g_{\star}^{1 / 2}\left(\frac{M_{W_{h}}}{10 \mathrm{GeV}}\right)^{3},
$$

which does not give an important limitation on $\varepsilon \cos \theta_{h}$ for scenarios of interest in our work, as we will see later. For $M_{\gamma_{h}} \gtrsim \mathrm{MeV}$, this also means that the $\gamma_{h}$ lifetime is much shorter than the Hubble time $\sim 1 \mathrm{~s}$ associated with big bang nucleosynthesis and $\gamma_{h}$ decays will not affect primordial nuclear processes.

The scalars in our model are expected to decay promptly. For example, given the assumption $M_{\Phi}>2 M_{W_{h}}$ above, $\Phi$ decays will be prompt, since they occur at tree-level and are not kinematically suppressed. The same applies to $\phi$ decays as long as $M_{\phi} \gtrsim M_{\gamma_{h}}$, since even for one off-shell $\gamma_{h}$, we expect a rate of order $\varepsilon^{2} g_{h}^{\prime 2} \alpha$ and for values of $\varepsilon$ that would be of interest here these decays will be prompt compared to the relevant cosmological time scales. 


\section{DIRECT DETECTION}

Another important quantity for a dark matter candidate is its direct-detection cross section. In the case of our $W_{h}$ candidate, this is governed by its scattering from protons in the nucleus by exchanging $Z_{h}$ or $\gamma_{h}$. Under our assumption $M_{\gamma_{h}} \ll M_{Z_{h}}$, the $\gamma_{h}$ exchange is the dominant contribution to the scattering process. ${ }^{4}$ The elastic scattering cross section from a nucleus $N$, with atomic number $Z$, is then given by

$$
\sigma_{\mathrm{el}} \simeq \frac{4 Z^{2} \alpha\left(\varepsilon \cos \theta_{h}\right)^{2}\left(g_{h} \sin \theta_{h}\right)^{2} \mu_{\mathrm{r}}^{2}\left(W_{h}, N\right)}{M_{\gamma_{h}}^{4}},
$$

where $\mu_{\mathrm{r}}(X, Y)=M_{X} M_{Y} /\left(M_{X}+M_{Y}\right)$ is the reduced mass of the system; $M_{N}=A m_{n}, A$ is the mass number, and $m_{n} \simeq$ $938 \mathrm{MeV}$ is the mass of a nucleon $n$. In the above, terms higher order in dark matter velocity are ignored and nuclear form factors have been set to unity (a good simplifying approximation). The elastic cross section per proton is then given by [44]

$$
\sigma_{p} \simeq \frac{4 \alpha\left(\varepsilon \cos \theta_{h}\right)^{2}\left(g_{h} \sin \theta_{h}\right)^{2} \mu_{\mathrm{r}}^{2}\left(W_{h}, n\right)}{M_{\gamma_{h}}^{4}} .
$$

The elastic cross section per nucleon is obtained from $\sigma_{n}=\left(Z^{2} / A^{2}\right) \sigma_{p}$.

In Fig. 2, we have plotted $\sigma_{p}$ versus $M_{W_{h}}$, assuming $W_{h}$ is dark matter for various values of $\varepsilon \cos \theta_{h}$ as a function of $M_{\gamma_{h}}$. For consistency, we have chosen values of parameters that would yield the correct relic abundance from Eq. (9) for each value of $M_{W_{h}}$. In this plot, we have also presented various relevant constraints on $\sigma_{p}$ at $90 \%$ C.L. from LUX [45], XENON10 [46], XENON100 [48], the combined CDMSII-Si data [10,49], and CDMSlite [50]. For illustration, the dot in the plot marks the maximumlikelihood point $\left(8.6 \mathrm{GeV}, \sigma_{n}=1.9 \times 10^{-41} \mathrm{~cm}^{2}, \sigma_{p}=\right.$ $7.6 \times 10^{-41} \mathrm{~cm}^{2}$ ) from the CDMSII-Si data [10]. We note that XENON10, XENON100, and LUX data disfavor this signal. However, there may be models that could lead to loosened constraints on this point in the parameter space $[51,52] .^{5}$

It is amusing to note that a lower bound on $M_{\gamma_{h}}$ can be obtained by combining an observation of DM at a direct

\footnotetext{
${ }^{4}$ Considering Higgs mixing, additional scattering channels may open. Scattering through $\Phi$ is suppressed compared to $\gamma_{h}$ under the assumption $M_{\Phi}>2 M_{W_{h}} \gg M_{\gamma_{h}}$. Additionally, scattering through $\phi$ is doubly suppressed by $\phi-\Phi$ and $\varphi-H$ mixing, which are expected to be small by arguments presented in Sec. III.

${ }^{5}$ After this paper was completed, the LUX results were released [45]. Although it appears that the minimal framework presented here cannot alleviate the tension between LUX and the CDMSII-Si maximum-likelihood point [10], we keep the latter only for illustrative purposes. We stress that the framework presented here is general and provides a valid model of DM in the allowed regions of parameter space.
}

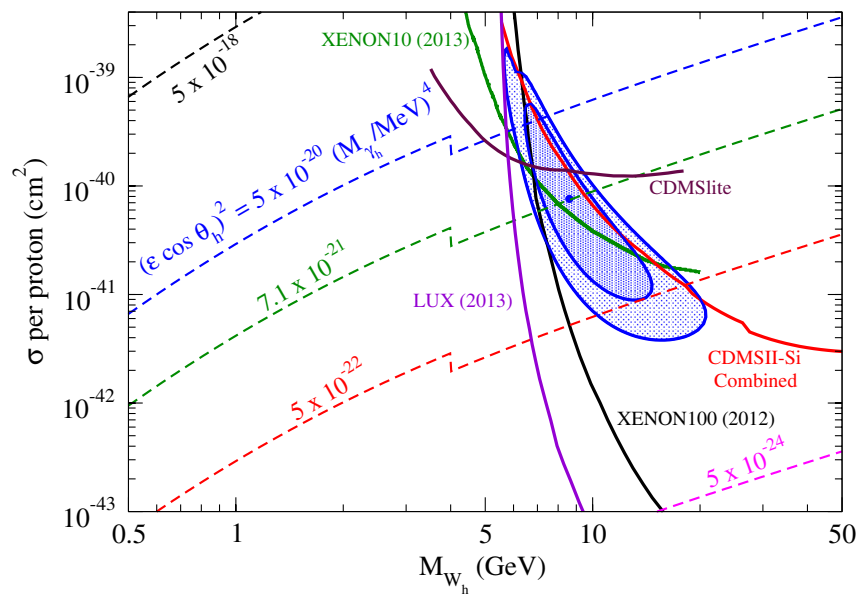

FIG. 2 (color online). The elastic cross section for various values of $\varepsilon \cos \theta_{h}$ as a function of $M_{W_{h}}$. Constraints from direct detection experiments are also included. For illustration, the dot corresponds to the CDMSII-Si highest-likelihood point [10]. The inner contour surrounding the dot is the $68 \%$ C.L. and the outer the $90 \%$ C.L.

detection experiment and the requirement that $\gamma_{h}$ remain in thermal equilibrium until $T_{f}$. Once $\sigma_{p}$ and $M_{W_{h}}$ are measured, Eq. (16) can be combined with the relic density constraint (11) to obtain a relation between $\varepsilon \sin \theta_{h}$ and $M_{\gamma_{h}}$. This relation can then be used in conjunction with Eq. (14) to obtain a lower bound on $M_{\gamma_{h}}$,

$$
\begin{aligned}
\frac{M_{\gamma_{h}}}{40 \mathrm{MeV}} \gtrsim & \left(\frac{M_{W_{h}}}{10 \mathrm{GeV}}\right)^{2 / 3}\left(\frac{\mu_{r}\left(W_{h}, n\right)}{1 \mathrm{GeV}}\right)^{1 / 3} \\
& \times\left(\frac{\sigma_{p}}{8 \times 10^{-41} \mathrm{~cm}^{2}}\right)^{-1 / 6}
\end{aligned}
$$

This lower bound was derived using the condition in Eq. (13) which is valid for $M_{\gamma_{h}}<T_{f}$ [43]. For $M_{W_{h}} \gtrsim$ $1 \mathrm{GeV}$ and $\sigma_{p} \gtrsim 10^{-43} \mathrm{~cm}^{2}$, the $M_{\gamma_{h}}$ lower bound given above is below $T_{f}$ and remains valid.

\section{FIXED TARGET AND DARK MATTER BEAM EXPERIMENTS}

There has been much interest in searching for new light weakly coupled vector bosons in fixed target and beamdump experiments [14-20]. In these experiments, when an electron scatters off the target it bremsstrahlungs a light gauge boson, which subsequently decays to a lepton pair. New light vector bosons can also be searched for in lowenergy $e^{+} e^{-}$experiments $[15,21]$ and in meson decays $[14,15,22-26]$. Such searches typically depend on finding resonances in the resulting dilepton spectrum.

The model presented here contains two vector bosons, $\gamma_{h}$ and $Z_{h}$, that couple to the SM electromagnetic current via kinetic mixing as shown in Eq. (7). Under our assumptions that $M_{Z_{h}} \geq 2 M_{W_{h}}$ we expect $Z_{h} \rightarrow W_{h} W_{h}$ to be the 
DARK MATTER FROM HIDDEN FORCES

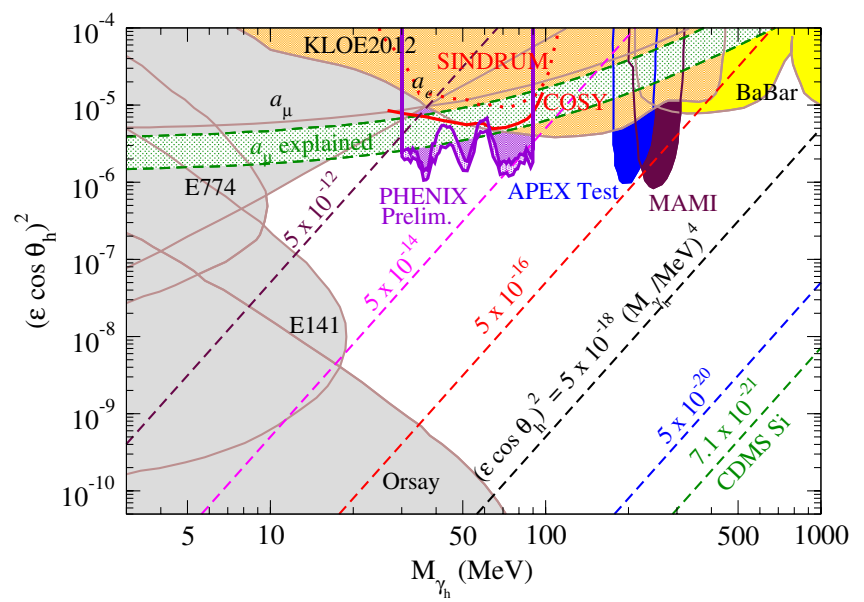

FIG. 3 (color online). Shaded regions indicate parameter space ruled out by various experiments. The shaded green region ( $a_{\mu}$ explained) indicates the parameter region that accommodates the muon $g_{\mu}-2$ anomaly. The line $\left(\varepsilon \cos \theta_{h}\right)^{2}=7.1 \times$ $10^{-21}\left(M_{\gamma_{h}} / \mathrm{MeV}\right)^{4}$ is consistent with the CDMSII-Si anomaly.

dominant decay channel since the $Z_{h}$ couplings to the SM are suppressed compared to the $Z_{h}-W_{h}$ coupling. However, with our assumptions, the only decay channels available for $\gamma_{h}$ is into light SM fermions. Hence, only $\gamma_{h}$ is expected to contribute to dilepton signals significantly.

In Fig. 3 we show the current bounds from low-energy $e^{+} e^{-}$experiments [15,21], meson decays ( $\pi^{0}$ at WASA-atCOSY [22], SINDRUM [23,24] and PHENIX [25]; $\phi$ at KLOE [26]; and $\Upsilon$ at BABAR [14,15]), fixed target experiments (MAMI [18] and APEX [19]), and the electron magnetic moment $[27,28]$. The shaded green region ( $a_{\mu}$ explained) indicates the parameter region consistent with the muon magnetic moment anomaly, $g_{\mu}-2$ [29-32]. We include dashed lines of parameter combinations that are relevant for the direct detection of $W_{h}$. The sensitivities to the $\varepsilon \cos \theta_{h}$ and $M_{\gamma_{h}}$ are to be compared to the direct detection experiment sensitivities in Fig. 2.

In Fig. 4 we show the sensitivity of the future fixed target experiments HPS, DarkLight, APEX, and VEPP3 [33]. For an overview of the HPS, DarkLight, and APEX experiments please see Ref. [34]. We note that the VEPP3 experiment is insensitive to the decay products of the light vector boson. Comparing to Fig. 2, it can be seen that these future experiments begin to be sensitive to $\varepsilon \cos \theta_{h}$ and $M_{\gamma_{h}}$ combinations that are relevant to light DM direct searches.

There are also proposals to measure invisibly decaying hidden bosons at fixed target experiments [53,54]. As before, hidden bosons are produced during interactions between the incident beam and the target. Subsequently, the hidden boson decays produce a beam of DM which then scatters off the nuclei of a detector, similar to direct detection experiments (however, here DM is relativistic). Typically it is assumed that the same hidden boson produces the DM and mediates the scattering off nuclei.
PHYSICAL REVIEW D 89, 055026 (2014)

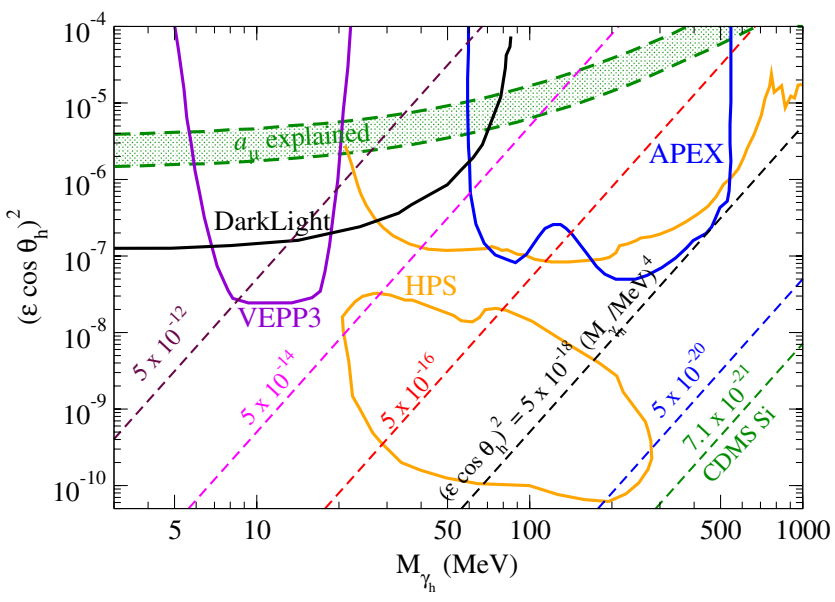

FIG. 4 (color online). Future sensitivities at fixed target experiments. The line corresponding to $\left(\varepsilon \cos \theta_{h}\right)^{2}=7.1 \times$ $10^{-21}\left(M_{\gamma_{h}} / \mathrm{MeV}\right)^{4}$ is consistent with the CDMSII-Si anomaly.

However, in the scenario presented here, the dark matter would be produced via $Z_{h}$ and then recoil dominantly via $\gamma_{h}$, since this is a $t$-channel process. We may hence expect the scenario presented here to manifest itself in many distinct experimental scenarios with tightly correlated parameter spaces.

\section{CONCLUSIONS}

In this work, we considered a hidden-sector gauge symmetry $\mathrm{SU}(2)_{h} \times \mathrm{U}(1)_{h}$, broken completely by a Higgs doublet of $\mathrm{SU}(2)_{h}$ and a Higgs singlet field charged only under $\mathrm{U}(1)_{h}$. Our assumed gauge sector provides a simple setup to illustrate possible effects of non-Abelian interactions that may govern the dynamics of DM. The hidden sector was only assumed to interact with the SM indirectly, through kinetic mixing of $\mathrm{U}(1)_{h}$ and hypercharge $\mathrm{U}(1)_{Y}$. This setup was shown to yield a good DM candidate, corresponding to hidden non-Abelian gauge fields, removing the need to assume extra fermions or scalars for DM. The stability of DM is guaranteed by a $Z_{2}$ remnant of the original gauge symmetry. Hence, in this framework DM is a manifestation of hidden or dark forces of nature. Our model can readily lead to the correct relic density and an allowed direct-detection cross section. In particular, the model can accommodate the recent tentative signal from CDMSII-Si [10] corresponding to DM masses $\sim 10 \mathrm{GeV}$.

Since our framework has an expanded hidden gauge sector, compared to models where only a dark U(1) is assumed, we find that the hidden and the SM sectors interact via the exchange of two distinct vectors bosons. These vector bosons are coupled to the electromagnetic current of the SM with a suppressed coupling governed by the degree of kinetic mixing. Typically, one would then expect signals corresponding to two different resonances that are often called "dark photons" in the U(1) models. 
Therefore, for light DM, the well-studied phenomenology of dark photons at low energies can be relevant to our model.

We also note that the two dark photons that arise in our model can be naturally hierarchic in mass and the heavier vector can in principle decay to vector DM. This process is mediated by an analogue of the SM $Z W^{+} W^{-}$vertex, where $\mathrm{DM}$ is the hidden counterpart to $W^{ \pm}$. In this case, the heavy vector is mainly invisible, since it would dominantly decay into DM, given its suppressed dark-photon-like coupling to the SM sector. Hence, our model can lead to "dark matter beam" signals in fixed target experiments that have been proposed in the context of U(1) models. In the typical DM beam scenario, the same vector that produces the DM is also responsible for DM scattering in the detector. In our setup, the DM is produced via the decay of the heavy vector while the detection is dominated by the exchange of the light vector. Thus, here the production and detection of the DM beam are mediated by two different vectors, the lighter of which is expected to have mainly visible decay modes.

We note that the light vectors of the hidden dynamics in our model may contribute to novel rare Higgs decay signals [56]. Also, present-day annihilation of $W_{h}$ in the Galactic halo may lead to potential $\mathrm{GeV}$-scale indirect detection signals. The totality of the signals available in this framework can in principle be used to discern the hidden gauge sector and its pattern of symmetry breaking, and potentially lead to the conclusion that "dark force = dark matter."

Finally, although the focus of this paper is on light dark matter scenarios, the framework presented here is more general. It can accommodate dark matter of most energy scales, including weakly interacting massive particle dark matter with masses of $\mathcal{O}(100 \mathrm{GeV})$.

\section{ACKNOWLEDGMENTS}

This work was supported in part by the United States Department of Energy under Grant Contracts DE-AC0298CH10886.

\section{APPENDIX A: MASS DIAGONALIZATION}

\section{Hidden sector}

The dark sector consists of two "charged" gauge bosons $W_{h}^{ \pm}$, and two "neutral" gauge bosons $W_{h}^{3}, B_{h}$. The masses of $W_{h}^{ \pm}$are then given simply by

$$
M_{W_{h}}=\frac{g_{h}}{2} v_{\Phi} .
$$

In the $\left(B_{h}, W_{h}^{3}\right)$ basis, the "neutral" mass matrix is

$$
\frac{1}{4}\left(\begin{array}{cc}
g_{h}{ }^{2}\left(v_{\Phi}^{2}+v_{\varphi}^{2}\right) & -g_{h} g_{h}{ }^{\prime} v_{\Phi}^{2} \\
-g_{h} g_{h}{ }^{\prime} v_{\Phi}^{2} & g_{h}^{2} v_{\Phi}^{2}
\end{array}\right) .
$$

The above matrix is then diagonalized via the transformation

$$
\left(\begin{array}{c}
B_{h} \\
W_{h}^{3}
\end{array}\right)=\left(\begin{array}{cc}
\cos \theta_{h} & -\sin \theta_{h} \\
\sin \theta_{h} & \cos \theta_{h}
\end{array}\right)\left(\begin{array}{l}
\gamma_{h}^{0} \\
Z_{h}^{0}
\end{array}\right),
$$

where $\gamma_{h}^{0}$ and $Z_{h}^{0}$ are mass eigenstates of the $2 \times 2$ matrix with masses $M_{\gamma_{h}^{0}}$ and $M_{Z_{h}^{0}}$, respectively, such that $M_{Z_{h}^{0}}>M_{\gamma_{h}^{0}}$.

In analogy with the SM weak mixing angle, we introduce a new angle completely determined by the hidden sector gauge couplings,

$$
\tan \zeta=g_{h}^{\prime} / g_{h} .
$$

The mass matrix in Eq. (A2) can then be written in the somewhat simpler form

$$
M_{W_{h}}^{2}\left(\begin{array}{ll}
\left(1+\eta^{2}\right) \tan ^{2} \zeta & -\tan \zeta \\
-\tan \zeta & 1
\end{array}\right),
$$

where, again, $\eta=v_{\phi} / v_{\Phi}$ is the ratio of the hidden sector Higgs VEVs. The masses for $\gamma_{h}^{0}$ and $Z_{h}^{0}$ can then be expressed as

$$
\begin{aligned}
M_{Z_{h}^{0}}^{2}+M_{\gamma_{h}^{0}}^{2} & =\frac{M_{W_{h}}^{2}}{\cos ^{2} \zeta}\left(1+\eta^{2} \sin ^{2} \zeta\right), \\
\frac{M_{Z_{h}^{0}}^{2}-M_{\gamma_{h}^{0}}^{2}}{M_{Z_{h}^{0}}^{2}+M_{\gamma_{h}^{0}}^{2}} & =\sqrt{1-\frac{\eta^{2} \sin ^{2} 2 \zeta}{\left(1+\eta^{2} \sin ^{2} \zeta\right)^{2}}} .
\end{aligned}
$$

The mixing angle $\theta_{h}$ can be solved for and we get

$$
\cos ^{2} \theta_{h}=\frac{M_{W_{h}}^{2}-M_{\gamma_{h}^{0}}^{2}}{M_{Z_{h}^{0}}^{2}-M_{\gamma_{h}^{0}}^{2}} .
$$

Since we have already assumed $M_{Z_{h}^{0}} \geq M_{\gamma_{h}^{0}}$, the positivity of $\cos ^{2} \theta_{h}$ enforces the hierarchy $M_{W_{h}} \geq M_{\gamma_{h}^{0}}$. Similarly, the positivity of $\sin ^{2} \theta_{h}$ can be used to show $M_{Z_{h}^{0}} \geq M_{W_{h}}$. We then naturally have the mass hierarchy $M_{Z_{h}^{0}} \geq M_{W_{h}} \geq M_{\gamma_{h}^{0}}$.

An interesting limit to look at is $\eta \ll 1$. From Eq. (A5), it is clear that the effect of the singlet Higgs $\phi$ is a mild perturbation in the usual SM neutral vector-boson mass matrix. Explicitly, we find

$$
\begin{gathered}
M_{\gamma_{h}^{0}}=M_{W_{h}} \eta \sin \zeta+\mathcal{O}\left(\eta^{3}\right), \\
M_{Z_{h}^{0}}=\frac{M_{W_{h}}}{\cos \zeta}\left(1+\frac{1}{2} \eta^{2} \sin ^{4} \zeta\right)+\mathcal{O}\left(\eta^{4}\right), \\
\tan \theta_{h}=\tan \zeta\left(1+\eta^{2} \sin ^{2} \zeta\right)+\mathcal{O}\left(\eta^{4}\right) .
\end{gathered}
$$

As expected, this is the same as the SM result plus small perturbations in $\eta$. In the limit $\eta \rightarrow 0$ the SM mass relations are exactly reproduced. 


\section{Kinetic mixing}

Now we consider the effects of kinetic mixing,

$$
\mathcal{L}_{\text {kin }}=-\frac{1}{4} \hat{B}_{\mu \nu} \hat{B}^{\mu \nu}+\frac{1}{2} \frac{\varepsilon}{\cos \theta_{W}} \hat{B}_{\mu \nu} \hat{B}_{h}^{\mu \nu}-\frac{1}{4} \hat{B}_{h \mu \nu} \hat{B}_{h}^{\mu \nu} .
$$

The kinetic terms are diagonalized via the shifts

$$
\begin{gathered}
\hat{B}^{\mu}=B^{\mu}+\frac{\varepsilon / \cos \theta_{W}}{\sqrt{1-\varepsilon^{2} / \cos ^{2} \theta_{W}}} B_{h}^{\mu}, \\
\hat{B}_{h}^{\mu}=\frac{1}{\sqrt{1-\varepsilon^{2} / \cos ^{2} \theta_{W}}} B_{h}^{\mu},
\end{gathered}
$$

where $B^{\mu}$ is now identified as the SM hypercharge and $B_{h}$ is identified as the $\mathrm{U}(1)_{h}$ gauge boson.

The transformation of $\hat{B}_{h}$ does not involve $B$ and so does not introduce any SM fields into the hidden-sector covariant derivative. However, the transformation of $\hat{B}$ does introduce $B_{h}$ into the SM covariant derivative. Hence, all induced mass mixing comes from the SM Higgs VEV and not the hidden sector. Specifically, after diagonalizing the kinetic term, the SM covariant derivative is

$$
\begin{aligned}
D_{\mu}^{\mathrm{SM}}= & \partial_{\mu}+i\left(\frac{g^{\prime} \xi}{\sin \theta_{W}} Y B_{h \mu}+g^{\prime} Y B_{\mu}+g T^{3} W_{\mu}^{3}\right. \\
& \left.+\frac{g}{\sqrt{2}}\left(\tau^{+} W_{\mu}^{+}+\tau^{-} W_{\mu}^{-}\right)\right),
\end{aligned}
$$

where $Y$ is the SM hypercharge operator, $T^{3}$ is the SM isospin operator, $\tau^{ \pm}$are the charged current operators, and $g$ and $g^{\prime}$ are the $\mathrm{SU}(2)$ and $\mathrm{U}(1)_{Y} \mathrm{SM}$ gauge couplings, respectively. The parameter

$$
\xi=\varepsilon \tan \theta_{W} / \sqrt{1-\varepsilon^{2} / \cos ^{2} \theta_{W}}
$$

has been introduced to simplify notation. Performing the usual SM rotation $B_{\mu}=\cos \theta_{W} A_{\mu}-\sin \theta_{W} \hat{Z}_{\mu}^{0}$ and $W_{\mu}^{3}=\sin \theta_{W} A_{\mu}+\cos \theta_{W} \hat{Z}_{\mu}^{0}$, as well as the rotation in Eq. (A3), the SM covariant derivative is

$$
\begin{aligned}
D_{\mu}^{\mathrm{SM}}= & \partial_{\mu}+i\left(\frac{g^{\prime} \xi}{\sin \theta_{W}} Y\left(\cos \theta_{h} \gamma_{h \mu}^{0}-\sin \theta_{h} Z_{h \mu}^{0}\right)\right. \\
& \left.+e Q A_{\mu}+g_{Z} Q_{Z} \hat{Z}_{\mu}^{0}+\frac{g}{\sqrt{2}}\left(\tau^{+} W_{\mu}^{+}+\tau^{-} W_{\mu}^{-}\right)\right),
\end{aligned}
$$

where $e$ and $Q=T^{3}+Y$ are the usual electromagnetic charge and operator; $g_{Z}=e /\left(\sin \theta_{W} \cos \theta_{W}\right)$ and $Q_{Z}=$ $T^{3} \cos ^{2} \theta_{W}-Y \sin ^{2} \theta_{W}$ are the SM neutral-current coupling and operator, respectively.

It is now clear that once the Higgs VEV is inserted into the Higgs kinetic term, there is mass mixing between the $\hat{Z}^{0}, \gamma_{h}^{0}$, and $Z_{h}^{0}$ proportional to the kinetic mixing parameter $\varepsilon$. In the $\left(\hat{Z}^{0}, \gamma_{h}^{0}, Z_{h}^{0}\right)$ basis, the mass matrix is

$$
M_{Z^{0}}^{2}\left(\begin{array}{ccc}
1 & -\xi \cos \theta_{h} & \xi \sin \theta_{h} \\
-\xi \cos \theta_{h} & \mu_{\gamma_{h}^{0}}^{2}+\xi^{2} \cos ^{2} \theta_{h} & -\xi^{2} \sin \theta_{h} \cos \theta_{h} \\
\xi \sin \theta_{h} & -\xi^{2} \sin \theta_{h} \cos \theta_{h} & \mu_{Z_{h}^{0}}^{2}+\xi^{2} \sin ^{2} \theta_{h}
\end{array}\right),
$$

where $M_{Z^{0}}=g_{Z} v / 2$ is the SM $Z$ mass and $\mu_{i}=M_{i} / M_{Z^{0}}$. To leading order in $\varepsilon$, the $3 \times 3$ mass matrix can be diagonalized with the transformations

$$
\begin{gathered}
\gamma_{h \mu}^{0} \simeq \gamma_{h \mu}-\frac{\varepsilon \tan \theta_{w} \cos \theta_{h}}{1-\mu_{\gamma_{h}^{0}}^{2}} Z_{\mu}^{0}, \\
Z_{h \mu}^{0} \simeq Z_{h \mu}+\frac{\varepsilon \tan \theta_{W} \sin \theta_{h}}{1-\mu_{Z_{h}^{0}}^{2}} Z_{\mu}^{0}, \\
\hat{Z}_{\mu}^{0} \simeq Z_{\mu}^{0}+\varepsilon \tan \theta_{W}\left(\frac{\cos \theta_{h}}{1-\mu_{\gamma_{h}^{0}}^{2}} \gamma_{h \mu}-\frac{\sin \theta_{h}}{1-\mu_{Z_{h}^{0}}^{2}} Z_{h \mu}\right),
\end{gathered}
$$

where $\gamma_{h}, Z_{h}$ and $Z^{0}$ are the mass eigenstates with masses

$$
\begin{gathered}
M_{\gamma_{h}}^{2} \simeq M_{\gamma_{h}^{0}}^{2}\left(1-\xi^{2} \frac{\cos ^{2} \theta_{h}}{1-\mu_{\gamma_{h}^{0}}^{2}}\right), \\
M_{Z_{h}}^{2} \simeq M_{Z_{h}^{0}}^{2}\left(1-\xi^{2} \frac{\sin ^{2} \theta_{h}}{1-\mu_{Z_{h}^{0}}^{2}}\right),
\end{gathered}
$$

$M_{Z}^{2} \simeq M_{Z^{0}}^{2} \times\left(1+\xi^{2} \frac{1-\mu_{Z_{h}^{0}}^{2} \cos ^{2} \theta_{h}-\mu_{\gamma_{h}^{0}}^{2} \sin ^{2} \theta_{h}}{\left(1-\mu_{\gamma_{h}^{0}}^{2}\right)\left(1-\mu_{Z_{h}^{0}}^{2}\right)}\right)$,

respectively. We have kept to $\mathcal{O}\left(\varepsilon^{2}\right)$ since this is the lowest order of $\varepsilon$ in Eqs. (A23-A25). Using these masses we see that Eq. (5) is valid to $\mathcal{O}\left(\varepsilon^{2}\right)$.

Finally, the interactions between the gauge bosons with fermions and scalars are governed by the covariant derivative. Applying the final transformation to the mass eigenstate, to $\mathcal{O}\left(\varepsilon^{2}\right)$ the SM covariant derivative is

$$
\begin{aligned}
D_{\mu}^{\mathrm{SM}}= & \partial_{\mu}+i \frac{g}{\sqrt{2}}\left(\tau^{+} W_{\mu}^{+}+\tau^{-} W_{\mu}^{-}\right)+i e Q A_{\mu}+i g_{Z} Q_{Z} Z_{\mu} \\
& +i \varepsilon e \cos \theta_{h}\left(Q+Q_{Z} \sec ^{2} \theta_{W} \frac{\mu_{\gamma_{h}^{0}}^{2}}{1-\mu_{\gamma_{h}^{0}}^{2}}\right) \gamma_{h \mu} \\
& \left.-i \varepsilon e \sin \theta_{h}\left(Q+Q_{Z} \sec ^{2} \theta_{W} \frac{\mu_{Z_{h}^{0}}^{2}}{1-\mu_{Z_{h}^{0}}^{2}}\right) Z_{h \mu} . \quad \text { A } 25\right)
\end{aligned}
$$

The interactions between $\gamma_{h}\left(Z_{h}\right)$ and the SM neutral current is suppressed by an additional factor of $\mu_{\gamma_{h}^{0}}^{2}\left(\mu_{Z_{h}^{0}}^{2}\right)$.

We also note that the transformations in Eqs. (A19) and (A20) will induce couplings between the SM $Z^{0}$ and the 
dark-sector currents. However, these currents are suppressed by $\varepsilon$ and not expected to make significant contributions to the electroweak precision observables.

\section{APPENDIX B: RELIC DENSITY CALCULATION}

For the reference of the reader, we briefly review the calculation of the relic density and obtain a general formula.

\section{Thermally averaged cross section}

The calculation of the thermally averaged cross section follows Ref. [55]. The general annihilation process

$$
\chi\left(p_{1}\right)+\chi\left(p_{2}\right) \rightarrow X\left(p_{X}\right)
$$

is considered, where $\chi$ is a DM candidate and $X$ is some final state that may be multiparticle. The usual annihilation cross section is then

$$
\sigma_{\mathrm{ann}} v_{\mathrm{rel}}=\frac{1}{4 E_{1} E_{2}} \int \overline{|\mathcal{M}(\chi \chi \rightarrow X)|^{2}} d P S_{X}
$$

where $v_{\text {rel }}$ is the relative speed of the DM particles, $\overline{\mid \mathcal{M}}^{2}$ is the spin summed and averaged matrix element squared, and $d P S_{X}$ is the final-state phase space.

The thermally averaged cross section is evaluated by performing a weighted integral over the possible initialstate momentum configurations,

$$
\left\langle\sigma_{\text {ann }} v_{\text {rel }}\right\rangle \equiv \frac{\kappa^{2}}{n_{\text {eq }}^{2}} \int \frac{d^{3} p_{1}}{(2 \pi)^{3}} \frac{d^{3} p_{2}}{(2 \pi)^{3}} f\left(E_{1}\right) f\left(E_{2}\right) \sigma_{\text {ann }} v_{\text {rel }},
$$

where $f(E)$ is the energy distribution of particle $\chi, \kappa$ is the number of internal degrees of freedom of $\chi$, and $n_{\mathrm{eq}}=$ $\kappa /(2 \pi)^{3} \int d^{3} p f(E)$ is the equilibrium density.

In general, $\sigma_{\text {ann }} v_{\text {rel }}$ is dependent upon the reference frame in which it is evaluated. Following Ref. [55], it is useful to introduce the Lorentz-invariant quantity

$$
w(s)=\frac{1}{4} \int \overline{|\mathcal{M}(\chi \chi \rightarrow X)|^{2}} d P S_{X} .
$$

After integration over the final-state phase space, the function $w$ can only depend on the particle masses and $s=\left(p_{1}+p_{2}\right)^{2}$. Hence, $w$ can be evaluated in a specific frame and then generalized to an arbitrary frame with the identification

$$
s=2\left(M_{\chi}^{2}+E_{1} E_{2}-p_{1} p_{2} \cos \theta_{12}\right),
$$

where $\theta_{12}$ is the angle between the initial-state momenta.

We are interested in temperatures such that $x=M_{\chi} / T \gg$ 1 and will express the final result as an expansion in $1 / x$. In this limit, the energy distributions are well approximated by the Boltzmann distribution $f(E)=\exp (-E / T)$. The integrals in Eq. (B3) are simplified by the change of variables

$$
y_{i}=\frac{E_{i}-M_{\chi}}{T}=\frac{E_{i}}{T}-x,
$$

for $i=1,2$. Using these variables, we can solve for

$$
\begin{aligned}
z \equiv \frac{s}{4 M_{\chi}^{2}}= & 1+\frac{1}{2 x}\left(y_{1}+y_{2}\right)+\frac{1}{2 x^{2}} y_{1} y_{2} \\
& -\frac{1}{x} \sqrt{\left(y_{1}+\frac{1}{2 x} y_{1}^{2}\right)\left(y_{2}+\frac{1}{2 x} y_{2}^{2}\right)} \cos \theta_{12} .
\end{aligned}
$$

Hence, an expansion in $1 / x$ can be obtained by expanding $w(s)$ around $s=4 M_{\chi}^{2}$ and the rest of the integrand around $1 / x$. The final result is

$$
\left\langle\sigma_{\mathrm{ann}} v_{\mathrm{rel}}\right\rangle=\left.\frac{1}{M_{\chi}^{2}}\left[w-\frac{3}{2 x}\left(2 w-w^{\prime}\right)+\mathcal{O}\left(x^{-2}\right)\right]\right|_{z=1},
$$

where $w^{\prime}=\partial w / \partial z$. The $\mathcal{O}\left(x^{0}\right)$ term is typically referred to as the $s$-wave and the $\mathcal{O}\left(x^{-1}\right)$ as the $p$-wave.

For completeness, we provide the full result of the thermally averaged cross section for $W_{h} W_{h} \rightarrow \gamma_{h} \gamma_{h}$, as shown in Fig. 1, up to the $p$-wave expansion,

$$
\begin{aligned}
\left\langle\sigma_{\mathrm{ann}} v_{\text {rel }}\right\rangle= & \frac{19\left(g_{h} \sin \theta_{h}\right)^{4}}{72 \pi M_{W_{h}}^{2}}\left(1-r_{\gamma_{h}}^{2}\right)^{-1 / 2}\left(1-\frac{r_{\gamma_{h}}^{2}}{2}\right)^{-4}\left[\left(1-\frac{55}{19} r_{\gamma_{h}}^{2}+\frac{295}{76} r_{\gamma_{h}}^{4}-\frac{241}{76} r_{\gamma_{h}}^{6}\right.\right. \\
& \left.+\frac{233}{152} r_{\gamma_{h}}^{8}-\frac{121}{304} r_{\gamma_{h}}^{10}+\frac{33}{608} r_{\gamma_{h}}^{12}-\frac{3}{608} r_{\gamma_{h}}^{14}\right) \\
& \left.-\frac{39}{19 x}\left(1-\frac{433}{156} r_{\gamma_{h}}^{2}+\frac{349}{78} r_{\gamma_{h}}^{4}-\frac{2851}{624} r_{\gamma_{h}}^{6}+\frac{7}{3} r_{\gamma_{h}}^{8}-\frac{5}{8} r_{\gamma_{h}}^{10}+\frac{77}{832} r_{\gamma_{h}}^{12}-\frac{41}{4992} r_{\gamma_{h}}^{14}\right)\right],
\end{aligned}
$$

where $r_{\gamma_{h}}=M_{\gamma_{h}} / M_{W_{h}}$. 


\section{a. Velocity expansion}

The above result has been explicitly constructed as an expansion of the thermal integral and $w$ in the variables $x=M_{\chi} / T$ and $z=s / 4 M_{\chi}^{2}$. Often one expands the annihilation cross section in terms of the relative velocity

$$
\sigma_{\mathrm{ann}} v_{\mathrm{rel}}=a+b v_{\mathrm{rel}}^{2} .
$$

Using the relation for the relative velocity

$$
v_{\text {rel }}^{2}=\left|\mathbf{v}_{\mathbf{1}}-\mathbf{v}_{\mathbf{2}}\right|^{2}=v_{1}^{2}+v_{2}^{2}-2 v_{1} v_{2} \cos \theta_{12},
$$

the thermally averaged cross section is then

$$
\left\langle\sigma_{\mathrm{ann}} v_{\mathrm{rel}}\right\rangle=a+\frac{6 b}{x},
$$

where $\mathbf{v}_{\mathbf{1 , 2}}$ are the velocities of the initial-state particles, and we have used the thermodynamic relation $\left\langle v_{\text {rel }}^{2}\right\rangle=6 / x$.

The advantage of using Eq. (B8) to calculate the thermally averaged cross section is that $w$ is Lorentz invariant and is expanded in terms of a Lorentz-invariant quantity. Hence, there are no frame-dependent ambiguities in calculating $\left\langle\sigma_{\mathrm{ann}} v_{\text {rel }}\right\rangle$. However, obtaining the usual result in Eq. (B12) from Eq. (B8) is not completely transparent. To illustrate how this is accomplished and that the two are equivalent, we now derive Eq. (B12) from Eq. (B8).

First we rewrite $w^{\prime}$ in Eq. (B8) as a derivative with respect to $v_{\text {rel }}^{2}$. Since $w$ is Lorentz invariant, this operation can be performed in the center-of-momentum frame,

$$
v_{\text {rel }}^{2}=4 v_{1}^{2}=4 v_{2}^{2}=4\left(1-\frac{1}{z}\right),
$$

where $v_{i}$ is the magnitude of the vector $\mathbf{v}_{\mathbf{i}}$. Hence, evaluating $w$ and its derivatives at $v_{\text {rel }}=0$ is equivalent to $z=1$.

For ease of notation, the velocity expansion of $w$ is expressed as

$$
w(s)=\alpha+\beta v_{\text {rel }}^{2},
$$

where $\alpha$ and $\beta$ are constants. The thermally averaged cross section is then

$$
\left\langle\sigma_{\text {ann }} v_{\text {rel }}\right\rangle=\frac{1}{M_{\chi}^{2}}\left[\alpha-\frac{3}{x}(\alpha-2 \beta)+\mathcal{O}\left(x^{-2}\right)\right] .
$$

To obtain Eq. (B12), $\alpha$ and $\beta$ need to be determined in terms of $a, b$. Since $\sigma_{\text {ann }} v_{\text {rel }}$ is not Lorentz invariant, we perform this in an arbitrary frame. That is we use the relative velocity in Eq. (B11) and the relation

$$
w(s)=E_{1} E_{2} \sigma_{\mathrm{ann}} v_{\text {rel }} .
$$

Expanding both sides to $\mathcal{O}\left(v_{1}^{2}, v_{2}^{2}, v_{1} v_{2}\right)$ and integrating over $\theta_{12}$, we find

$$
\alpha=M_{\chi}^{2} a, \quad \text { and } \quad \beta=M_{\chi}^{2}\left(b+\frac{a}{2}\right) .
$$

Using these results and Eq. (B8), we obtain the well-known result in Eq. (B12).

\section{Relic density}

The relic density of a particle $\chi$ is typically given in terms of the variable $\Omega=\rho(0) / \rho_{\text {crit }}$, where $\rho(0)$ is the present energy density of particle $\chi$. The critical density is $\rho_{\text {crit }}=$ $3 H^{2}(0) / 8 \pi G$ with $G$ being Newton's constant, $H(T)=$ $\dot{R} / R$ the expansion rate of the universe, and $R$ the cosmic scale factor. Since the relic $\chi$ is massive, at present day $x \gg 1$ and the energy density is $\rho(0)=M_{\chi} n(0)$, where $n(T)$ is the number density at temperature $T$. Hence, the number density needs to be solved for. The details of this derivation are well known and can, for example, be found in Refs. [43,55].

The number density, $n$, of particle $\chi$ obeys the Boltzmann equation

$$
\frac{d n}{d t}=-3 H(T) n-\left\langle\sigma_{\mathrm{ann}} v_{\mathrm{rel}}\right\rangle\left(n^{2}-n_{\mathrm{eq}}^{2}\right) .
$$

Using well-known methods [43,55], this differential equation can be simplified to

$$
\frac{d Y}{d x}=-\frac{\left\langle\sigma_{\mathrm{ann}} v_{\mathrm{rel}}\right\rangle s(T)}{x H(T)}\left(Y^{2}-Y_{\mathrm{eq}}^{2}\right),
$$

where $Y=n / s$ and $s$ is the entropy density.

To evaluate $n(0)$, late-time values of $x \gg 1$ need to be considered. After freeze-out, the number density $n$ is stable while the equilibrium number density $n_{\mathrm{eq}}$ continues to decrease as the photon temperature continues to drop. Hence we have $Y \gg Y_{\text {eq }}$ and the differential equation simplifies to

$$
\frac{d Y}{d x} \simeq-\frac{\left\langle\sigma_{\mathrm{ann}} v_{\mathrm{rel}}\right\rangle s(T)}{x H(T)} Y^{2} .
$$

To solve this equation we expand the thermally averaged cross section,

$$
\left\langle\sigma_{\mathrm{ann}} v_{\mathrm{rel}}\right\rangle=\sum_{j} a_{j} x^{-j}
$$

Furthermore, the effective degrees of freedom are introduced,

$$
g_{\star s}(T)=\frac{45}{2 \pi^{2}} \frac{s(T)}{T^{3}}, \quad g_{\star}(T)=\frac{30}{\pi^{2}} \frac{\rho(T)}{T^{4}},
$$


and the solution for $H$ in the Robertson-Walker metric is used,

$$
H(T)=\left(\frac{8 \pi}{3} G \rho(T)\right)^{1 / 2}
$$

Equation (B20) is then integrated from freeze-out, $x=x_{f}$, to present day, $x=+\infty$, to find

$$
Y_{\infty}=\left(\frac{45}{\pi}\right)^{1 / 2} \frac{1}{M_{\mathrm{Pl}} M_{\chi}} \frac{1}{g_{\star}^{-1 / 2} g_{\star s}} \frac{x_{f}}{\sum_{j} a_{j} x_{f}^{-j} /(j+1)},
$$

where $Y_{\infty}$ is the present-day value and $g_{\star}$ and $g_{\star s}$ are evaluated at freeze-out.

Finally, putting everything together, the relic density is

$$
\Omega h^{2}=\frac{5.36 \times 10^{43} \mathrm{~cm}^{3} \mathrm{GeV} s(0)}{M_{\mathrm{Pl}}^{3} g_{\star}^{-1 / 2} g_{\star s}} \frac{x_{f}}{\sum_{j} a_{j} x_{f}^{-j} /(j+1)},
$$

where $H(0)=\left(100 \mathrm{~km} \mathrm{~s}^{-1} \mathrm{Mpc}\right) h$ and $G=1 / M_{\mathrm{Pl}}^{2}$ have been used, and the present-day entropy density is $s(0)=$ $2889.2 \mathrm{~cm}^{-3}$ [42]. To a good approximation, at freeze-out $g_{\star} \simeq g_{\star S}$ and we use this in the numerical solutions presented in the text.
[1] P. A. R. Ade et al. (Planck Collaboration), arXiv:1303.5076.

[2] O. Adriani et al. (PAMELA Collaboration), Nature (London) 458, 607 (2009). See also Refs. [3,4]

[3] M. Ackermann et al. (Fermi LAT Collaboration), Phys. Rev. Lett. 108, 011103 (2012).

[4] M. Aguilar et al. (AMS Collaboration), Phys. Rev. Lett. 110, 141102 (2013).

[5] N. Arkani-Hamed, D. P. Finkbeiner, T. R. Slatyer, and N. Weiner, Phys. Rev. D 79, 015014 (2009).

[6] R. Bernabei et al. (DAMA and LIBRA Collaborations), Eur. Phys. J. C 67, 39 (2010).

[7] C. E. Aalseth et al. (CoGeNT Collaboration), Phys. Rev. D 88, 012002 (2013).

[8] G. Angloher et al., Eur. Phys. J. C 72, 1971 (2012); A. Brown, S. Henry, H. Kraus, and C. McCabe, Phys. Rev. D 85, 021301 (2012).

[9] F. Petriello and K. M. Zurek, J. High Energy Phys. 09 (2008) 047; C. Savage, G. Gelmini, P. Gondolo, and K. Freese, J. Cosmol. Astropart. Phys. 04 (2009) 010; A. L. Fitzpatrick, D. Hooper, and K. M. Zurek, Phys. Rev. D 81, 115005 (2010); S. Chang, J. Liu, A. Pierce, N. Weiner, and I. Yavin, J. Cosmol. Astropart. Phys. 08 (2010) 018; V. Barger, M. McCaskey, and G. Shaughnessy, Phys. Rev. D 82, 035019 (2010); W.-Y. Keung, I. Low, and G. Shaughnessy, Phys. Rev. D 82, 115019 (2010); M. R. Buckley, D. Hooper, and T. M. P. Tait, Phys. Lett. B 702, 216 (2011); K.-Y. Choi and O. Seto, Phys. Rev. D 88, 035005 (2013); R. C. Cotta, A. Rajaraman, T. M. P. Tait, and A. M. Wijangco, arXiv:1305.6609 [Phys. Rev. D (to be published)]; M. McCullough and L. Randall, J. Cosmol. Astropart. Phys. 10 (2013) 058.

[10] R. Agnese et al. (CDMS Collaboration), Phys. Rev. Lett. 111, 251301 (2013).

[11] For example of DM models based on such considerations see R. Foot, Int. J. Mod. Phys. D 13, 2161 (2004); M. Cirelli, N. Fornengo, and A. Strumia, Nucl. Phys. B753, 178 (2006); M. Pospelov, A. Ritz, and M. B. Voloshin, Phys. Lett. B 662, 53 (2008).
[12] T. Hambye, J. High Energy Phys. 01 (2009) 028; T. Hambye and M.H. G. Tytgat, Phys. Lett. B 683, 39 (2010); J. L. Diaz-Cruz and E. Ma, Phys. Lett. B 695, 264 (2011); S. Bhattacharya, J. L. Diaz-Cruz, E. Ma, and D. Wegman, Phys. Rev. D 85, 055008 (2012); F. D’Eramo, M. McCullough, and J. Thaler, J. Cosmol. Astropart. Phys. 04 (2013) 030; T. Hambye and A. Strumia, Phys. Rev. D 88, 055022 (2013). C. D. Carone and R. Ramos, Phys. Rev. D 88, 055020 (2013).

[13] C.-W. Chiang, T. Nomura, and J. Tandean, arXiv:1306.0882.

[14] J. D. Bjorken, R. Essig, P. Schuster, and N. Toro, Phys. Rev. D 80, 075018 (2009).

[15] M. Reece and L.-T. Wang, J. High Energy Phys. 07 (2009) 051 .

[16] S. Andreas, C. Niebuhr, and A. Ringwald, Phys. Rev. D 86, 095019 (2012).

[17] F. Bossi, in KLOE-2 Physics Workshop, Frascati, Italy, April 9-10, 2009 (unpublished); P.-f. Yin, J. Liu, and S.-h. Zhu, Phys. Lett. B 679, 362 (2009).

[18] H. Merkel et al. (A1 Collaboration), Phys. Rev. Lett. 106, 251802 (2011).

[19] S. Abrahamyan et al. (APEX Collaboration), Phys. Rev. Lett. 107, 191804 (2011).

[20] S. N. Gninenko, Phys. Lett. B 713, 244 (2012); arXiv:1308.6521 [Phys. Rev. D (to be published)].

[21] R. Essig, P. Schuster, and N. Toro, Phys. Rev. D 80, 015003 (2009); B. Batell, M. Pospelov, and A. Ritz, Phys. Rev. D 79, 115008 (2009); B. Batell, M. Pospelov, and A. Ritz, Phys. Rev. D 80, 095024 (2009).

[22] P. Adlarson et al. (WASA-at-COSY Collaboration), Phys. Lett. B 726, 187 (2013).

[23] S. N. Gninenko, Phys. Rev. D 87, 035030 (2013).

[24] R. M. Drees et al. (SINDRUM I Collaboration), Phys. Rev. Lett. 68, 3845 (1992).

[25] Y. Yamaguchi on behalf of the PHENIX Collaboration, in Proceedings of the 2013 Fall Meeting of the APS Division of Nuclear Physics, Newport News, VA, October 23-26, 2013 (to be published). 
[26] D. Babusci et al. (KLOE-2 Collaboration), Phys. Lett. B 720, 111 (2013).

[27] H. Davoudiasl, H.-S. Lee, and W. J. Marciano, Phys. Rev. D 86, 095009 (2012).

[28] M. Endo, K. Hamaguchi, and G. Mishima, Phys. Rev. D 86, 095029 (2012)

[29] P. Fayet, Phys. Rev. D 75, 115017 (2007).

[30] M. Pospelov, Phys. Rev. D 80, 095002 (2009).

[31] H. Davoudiasl, H.-S. Lee, and W. J. Marciano, Phys. Rev. Lett. 109, 031802 (2012).

[32] G. W. Bennett et al. (Muon G-2 Collaboration), Phys. Rev. D 73, 072003 (2006).

[33] B. Wojtsekhowski, D. Nikolenko, and I. Rachek, arXiv:1207.5089.

[34] J. R. Boyce (LIPSS, DarkLight, HPS and APEX Collaborations), J. Phys. Conf. Ser. 384, 012008 (2012).

[35] H. Davoudiasl, H.-S. Lee, and W. J. Marciano, Phys. Rev. D 85, 115019 (2012).

[36] H.-C. Cheng, K. T. Matchev, and M. Schmaltz, Phys. Rev. D 66, 036005 (2002); G. Servant and T. M. P. Tait, Nucl. Phys. B 650, 391 (2003); H.-C. Cheng, J. L. Feng, and K. T. Matchev, Phys. Rev. Lett. 89, 211301 (2002); D. Hooper and G. D. Kribs, Phys. Rev. D 70, 115004 (2004).

[37] H.-C. Cheng and I. Low, J. High Energy Phys. 09 (2003) 051; J. High Energy Phys. 08 (2004) 061; A. Birkedal, A. Noble, M. Perelstein, and A. Spray, Phys. Rev. D 74, 035002 (2006).

[38] O. Lebedev, H. M. Lee, and Y. Mambrini, Phys. Lett. B 707, 570 (2012); T. Abe, M. Kakizaki, S. Matsumoto, and O. Seto, Phys. Lett. B 713, 211 (2012); Y. Farzan and A. R. Akbarieh, J. Cosmol. Astropart. Phys. 10 (2012) 026; S. Baek, P. Ko, W.-I. Park, and E. Senaha, J. High Energy Phys. 05 (2013) 036; K.-Y. Choi, H. M. Lee, and O. Seto, Phys. Rev. D 87, 123541 (2013).

[39] B. Holdom, Phys. Lett. 166B, 196 (1986).

[40] Some recent works that employ kinetic mixing between the Standard Model and dark gauge sector include S. Gopalakrishna, S. Jung, and J. D. Wells, Phys. Rev. D 78, 055002 (2008); A. Hook, E. Izaguirre, and J. G. Wacker, Adv. High Energy Phys. 2011, 859762 (2011); Y. Mambrini, J. Cosmol. Astropart. Phys. 09 (2010) 022; Y. Mambrini, J. Cosmol. Astropart. Phys. 07 (2011) 009; A. Alves, E. R. Barreto, A. G. Dias, C. A. de S. Pires, F. S. Queiroz, and P. S. Rodrigues da Silva, Phys. Rev. D 84, 115004 (2011); A. Alves, A. G. Dias, E. Ramirez Barreto, C. A. de S.Pires, F. S.Queiroz, and P. S. Rodrigues da Silva, Eur. Phys. J. C 73, 2288 (2013).
[41] For discussions of non-Abelian dark gauge sectors, see M. Baumgart, C. Cheung, J. T. Ruderman, L.-T. Wang, and I. Yavin, J. High Energy Phys. 04 (2009) 014; F. Chen, J. M. Cline, and A. R. Frey, Phys. Rev. D 80, 083516 (2009); H. Zhang, C. S. Li, Q.-H. Cao and Z. Li, Phys. Rev. D 82, 075003 (2010); C. D. Carone, Phys. Lett. B 721, 118 (2013); D. G. E. Walker, arXiv:1202.2348.

[42] J. Beringer et al. (Particle Data Group Collaboration), Phys. Rev. D 86, 010001 (2012).

[43] E. Kolb and M. Turner, The Early Universe (Westview Press, Boulder, CO, 1990).

[44] R. Bernabei et al., Phys. Lett. B 389, 757 (1996); M. Pospelov and T. ter Veldhuis, Phys. Lett. B 480, 181 (2000).

[45] D. S. Akerib et al. (LUX Collaboration), arXiv:1310.8214 [Phys. Rev. Lett. (to be published)].

[46] J. Angle et al. (XENON10 Collaboration), Phys. Rev. Lett. 107, 051301 (2011); J. Angle et al., Phys. Rev. Lett. 110, 249901(E) (2013). See also Ref. [47].

[47] M. T. Frandsen, F. Kahlhoefer, C. McCabe, S. Sarkar, and K. Schmidt-Hoberg, J. Cosmol. Astropart. Phys. 07 (2013) 023.

[48] E. Aprile et al. (XENON100 Collaboration), Phys. Rev. Lett. 109, 181301 (2012).

[49] R. Agnese et al. (CDMS Collaboration), Phys. Rev. D 88, 031104 (2013).

[50] R. Agnese et al., Phys. Rev. Lett. 112, 041302 (2014).

[51] D. Hooper, J. Cosmol. Astropart. Phys. 09 (2013) 035.

[52] G. Belanger, A. Goudelis, J.-C. Park and A. Pukhov, arXiv:1311.0022; M. I. Gresham and K. M. Zurek, Phys. Rev. D 89, 016017 (2014); E. Del Nobile, G. B. Gelmini, P. Gondolo, and J.-H. Huh, arXiv:1311.4247; V. Cirigliano, M. L. Graesser, G. Ovanesyan, and I. M. Shoemaker, arXiv:1311.5886.

[53] B. Batell, M. Pospelov, and A. Ritz, Phys. Rev. D 80, 095024 (2009); P. deNiverville, M. Pospelov, and A. Ritz, Phys. Rev. D 84, 075020 (2011); P. deNiverville, D. McKeen and A. Ritz, Phys. Rev. D 86, 035022 (2012); R. Dharmapalan et al. (MiniBooNE Collaboration), arXiv:1211.2258.

[54] E. Izaguirre, G. Krnjaic, P. Schuster, and N. Toro, Phys. Rev. D 88, 114015 (2013); M. D. Diamond and P. Schuster, Phys. Rev. Lett. 111, 221803 (2013).

[55] M. Srednicki, R. Watkins, and K. A. Olive, Nucl. Phys. B310, 693 (1988).

[56] H. Davoudiasl, H.-S. Lee, I. Lewis, and W. J. Marciano, Phys. Rev. D 88, 015022 (2013). 